\title{
A IMPORTÂNCIA DO USO DE FILTROS, DURANTE A INFUSÃO DE NUTRIÇÕES PARENTERAIS ADICIONADAS DE EMULSÕES LIPÍDICAS
}

\author{
THE IMPORTANCE OF THE USE OF FILTERS DURING THE INFUSION OF \\ PARENTERAL NUTRITION CONTAINING LIPID EMULSIONS
}

Luiz M. Sakamoto ${ }^{\text {; }}$ Sírlei T. de Alcântara²; Nilva M. R. R. S. Passos ${ }^{3}$; Júlia K. S. Hotta ${ }^{4}$ \& Júlio S. Marchini ${ }^{5}$

\begin{abstract}
${ }^{1}$ Docente. Diretor do Serviço de Atividades Industriais; ${ }^{2}$ Diretora do Serviço de Dispensação e Distribuição. ${ }^{3}$ Docente, Chefe de Seção. Departamento de Ciências Farmacêuticas da Universidade de Ribeirão Preto. Divisão de Assistência Farmacêutica do HCFMRP, ${ }^{4}$ Biologista Encarregada do Laboratório de Nutrição do HCFMRP, ${ }^{5}$ Docente do Departamento de Clínica Médica - Divisão de Nutrição Clínica da Faculdade de Medicina de Ribeirão Preto da Universidade de São Paulo.

CorRespondência: Luiz Maçao Sakamoto - Serviço de Atividades Industriais da Divisão de Assistência Farmacêutica do HCFMRP-USP Campus Universitário Monte Alegre - Ribeirão Preto - SP - CEP 14048-900 - Fax: (0xx16) 633 - 0861.
\end{abstract}

SAKAMOTO LM; ALCÂNTARA ST; PASSOS NMRRS; HOTTA JKS \& MARCHINI JS. A importância do uso de filtros, durante a infusão de nutrições parenterais adicionadas de emulsões lipídicas. Medicina, Ribeirão Preto, 32:478-485; out./dez. 1999.

RESUMO: Nutrições parenterais com adicionamento de emulsões lipídicas podem apresentar alterações físico-químicas, caracterizadas pela formação de precipitados amorfos ou cristalinos, bem como pelo aumento do tamanho das gotículas de gordura que se agregam. A verificação macroscópica dessas alterações nem sempre é possível, tendo em vista o aspecto opaco das emulsões.

Utilizando-se estudo in vitro, este trabalho teve como objetivo evidenciar a presença de precipitados de fosfato de cálcio, de gotículas de gordura da emulsão lipídica com diâmetros superiores aos de hemácias, em nutrições parenterais, e a importância do uso de filtros na retenção dos mesmos. Esses eventos foram demonstrados por meio de inspeção macroscópica e microscópica, em nutrições parenterais contendo fosfato dibásico de potássio e de gluconato de cálcio, como fonte de fósforo e de cálcio, respectivamente, adicionados à mistura de soluções de aminoácidos a $10 \%$ p/v, com glicose a $50 \%$ p/v, emulsão lipídica a $20 \%$ p/v e minerais em diferentes proporções. Os resultados mostraram presença de precipitado de cálcio e de gotículas de gordura aumentadas e/ou agregadas antes da filtração, e ausência dos mesmos imediatamente após.

Conclusão: o uso de filtros com poros de 1,2 $\mu \mathrm{m}$ foi capaz de reter precipitados e de impedir a passagem das gotículas de gordura com dimensões tais que pudessem causar embolias microvasculares em pacientes submetidos à nutrição parenteral.

UNITERMOS: Filtração. Nutrição Parenteral. Emulsões.

\section{INTRODUÇÃO}

O suporte nutricional via parenteral é considerado instrumento médico fundamental para o restabelecimento nutricional em diferentes situações clínicas tais como: síndrome do intestino curto, fístulas in- testinais de alto débito, pancreatite aguda, desnutrição grave, grandes traumas, tratamento de prematuros, etc. . $^{(1,2,3)}$

De maneira simplificada ${ }^{(4)}$, nutrições parenterais em sistema glicídico, conhecidas como misturas $2 \mathrm{em}$ 1, são aquelas que contêm aminoácidos, glicose, vi- 
taminas e sais minerais em quantidades adequadas para satisfazer as necessidades diárias de um paciente específico. Quando acrescidas de emulsões lipídicas, em um mesmo recipiente, são conhecidas como misturas $3 \mathrm{em} \mathrm{1,} \mathrm{ou} \mathrm{sistema} \mathrm{lipídico.} \mathrm{Essas} \mathrm{misturas} \mathrm{são}$ preparações extemporâneas e devem ser completamente infundidas por períodos de, no máximo, 48 horas após o preparo ${ }^{(5)}$. No entanto, o uso desses nutrientes na recuperação dos pacientes não é isento de dificuldades e/ou incompatibilidades físico-químicas ${ }^{(6)}$. Entre as complicações de diversas naturezas ${ }^{(1,2,3)}$, pode-se citar osmolaridade elevada, sobrecarga de nutrientes, resultando em distúrbios metabólicos, falta de nutrientes, em especial dos micronutrientes ainda não disponibilizados pela indústria farmacêutica nacional, contaminações microbiológicas, formação de precipitados, incompatibilidades entre soluções aquosas e emulsões lipídicas, resultando em perda de estabilidade e, conseqüentemente, em agregação e aumento do tamanho das gotículas de gordura, dentre outras. Muitas dessas complicações, relacionadas com o preparo, podem ser evitadas, seguindo-se as Normas de Boas Práticas de Preparação de Nutrição Parenteral (BPPNP) e de Boas Práticas de Administração de Nutrição Parenteral (BPANP), recomendadas pelo Ministério da Saúde ${ }^{(5)}$. Por outro lado, o uso, cada vez mais freqüente, do suporte nutricional parenteral e o surgimento de novas exigências, como a adição crescente de diferentes sais e a utilização de soluções acrescidas de lipídios, ampliam a magnitude dos problemas relacionados a precipitações e a incompatibilidades físico-químicas das soluções em relação a diferentes tipos de fármacos.

A formação de precipitados insolúveis, como os de fosfato de cálcio ${ }^{(6 / 9)}$, pode ocorrer, quando se utilizam sais de cálcio, orgânicos ou inorgânicos, juntamente com sais de fosfatos inorgânicos. Esses precipitados são amorfos ou cristalinos, podendo surgir imediatamente, ou mesmo, horas após o preparo da nutrição parenteral. $\mathrm{O}$ aumento do $\mathrm{pH}$, o da temperatura e o da concentração dos diferentes solutos, na solução aquosa ou na emulsão lipídica, podem favorecer a formação de precipitados, bem como provocar a instabilidade físico-química do produto final. A presença dos precipitados é facilmente visualizada, quando a nutrição parenteral não contém lipídios. A simples turvação contra-indica, formalmente, o seu uso clínico. Nas formulações em sistema 3 em 1, a opacidade, provocada pela presença de lipídios, inviabiliza a verificação de qualquer turvação a olho nu.
Em adição, nutrições parenterais, contendo emulsões lipídicas, podem apresentar dois graves problemas: O primeiro é relacionado com o crescimento e/ ou a agregação das gotículas de gordura. O segundo, com a formação de precipitados de fosfato de cálcio, amorfos, ou cristalinos. Esses eventos podem ocorrer imediatamente ou horas após o preparo, podendo levar à insuficiência respiratória por embolia microvascular pulmonar difusa, em pacientes recebendo suporte nutricional via parenteral ${ }^{(10,11,12)}$.

Portanto, em consequiência do fato de que agregados de gotículas de gordura, ao lado de precipitados amorfos, podem ser formados durante o preparo das nutrições parenterais, o presente trabalho teve por objetivo mostrar a importância do uso de filtros com poros de 1,2 $\mu \mathrm{m}$ de diâmetro, durante a infusão. Esses filtros impedem que tais agregados, ou precipitados, sejam infundidos, endovenosamente, no paciente que recebe nutrição parenteral, evitando a ocorrência de possíveis efeitos colaterais.

Dessa forma, a hipótese do presente trabalho é que a filtração, imediatamente após o preparo das nutrições parenterais, elimina, definitivamente, a possibilidade da infusão de precipitados de fosfato de cálcio e de gotículas de gordura aumentadas ou agregadas.

\section{MATERIAIS E MÉTODOS}

O presente trabalho foi realizado na Divisão de Assistência Farmacêutica do Hospital das Clínicas da Faculdade de Medicina de Ribeirão Preto da Universidade de São Paulo (DAFHCFMRP-USP) e no Departamento de Farmácia da Universidade de Ribeirão Preto (DFURP). Para tanto, foram utilizados aparelho de Fluxo Laminar Horizontal, marca Veco®; bomba de infusão, marca JMS $® ;$ microscópio óptico, mar-

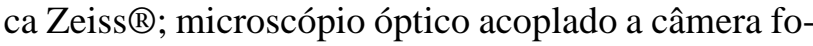
tográfica, marca Nikkon ${ }^{\circledR}$; suporte de filtro e membrana filtrante, com poros de $1,2 \mu \mathrm{m}$ e de diâmetro de $47 \mathrm{~mm}$, marca Millipore ${ }^{\circledR}$; bolsa de etileno acetato de vinila (EVA), marca Laboratórios B. Braun S/A®; vidrarias e seringas. Também foram utilizadas as seguintes soluções farmacológicas: aminoácidos, marca Laboratórios B. Braun S/A®; glicose, marca Serviço de Atividades Industriais do HCFMRP®; cloreto de sódio, marca Darrow ${ }^{\circledR}$; cloreto de potássio, marca Darrow ${ }^{\circledR}$; gluconato de cálcio, marca Ariston®; sulfato de magnésio, marca Ariston ${ }^{\circledR}$; fosfato dibásico de potássio, marca Serviço de Atividades Industriais 
do HCFMRP®; oligoelementos, marca Darrow ${ }^{\circledR}$; cloreto de cálcio, marca Serviço de Atividades Industriais do HCFMRP®; emulsão lipídica a $20 \% \mathrm{v} / \mathrm{v}$, marca B. Brawn ${ }^{\circledR}$ e água destilada, marca JP Indústria Farmacêutica $S / A \circledast$, rotineiramente utilizados pela DAFHCFMRP-USP.

Inicialmente, foram misturadas diferentes proporções, em mmol, de fosfato dibásico de potássio com cloreto de cálcio (2:1, 1:1 e 1:2), utilizando-se água destilada para um volume final de $100 \mathrm{~mL}$. Posteriormente, os ensaios foram repetidos com misturas de fosfato dibásico de potássio com gluconato de cálcio nas mesmas proporções. Também foram preparadas formulações em sistema lipídico, utilizando-se emulsão lipídica a $20 \%$ p/v, alterada devido à quebra da estabilidade após manutenção a $37^{\circ} \mathrm{C}$, durante quatro horas, com e sem fosfato (Tabela 1), na presença de gluconato de cálcio, de acordo com as normas técnicas recomendadas $^{(5)}$. Em seguida, as diferentes preparações de nutrições parenterais foram transferidas para bolsas de EVA e, com o auxílio de uma bomba de infusão, foram filtradas em membranas com poros de 1,2 $\mu \mathrm{m}$ de diâmetro a $50 \mathrm{~mL} / \mathrm{hora}$. Em todos esses procedimentos, foram seguidas recomendações apropriadas ${ }^{(5)}$.
A verificação de agregados de gotículas de lipídios e de cristais amorfos e/ou cristalinos de fosfato de cálcio foi feita por técnica microscópica a fresco, utilizando-se lâmina e lamínula em aumento de 400 e 1000 vezes. A verificação dos precipitados e agregados de lipídios foi feita nos tempos trinta minutos, uma hora, duas horas e 24 horas após o preparo.

\section{RESULTADOS}

Todas as misturas de fosfato dibásico de potássio com cloreto de cálcio e com gluconato de cálcio resultaram em precipitados de fosfato de cálcio, amorfos ou cristalinos, insolúveis (Figuras 1-A e 1-B), caracterizados, visualmente, pela turvação ou precipitação, em tempo não superior a uma hora. Observou-se que grande parte dos precipitados ficaram retidos nas bolsas de EVA, enquanto o restante, no filtro. Consequientemente, os filtrados se apresentaram límpidos, incolores e sem a presença de precipitados, após visualização macroscópica e microscópica. No entanto, quando mantidos à temperatura ambiente por 24 horas, verificou-se a formação de novos precipitados.

\begin{tabular}{|c|c|c|c|c|c|c|c|}
\hline \multirow{2}{*}{ Formulações * } & 1 & 2 & 3 & 4 & 5 & 6 & 7 \\
\hline & \multicolumn{7}{|c|}{$\mathrm{mL}$} \\
\hline Aminoácidos a 10\% p/v (pediátrico) & 5,0 & 8,5 & 17,0 & 25,0 & 12,0 & 20,0 & 30,0 \\
\hline Glicose a $50 \%$ p/v & 15,0 & 15,0 & 20,0 & 25,0 & 16,0 & 24,0 & 30,0 \\
\hline Emulsão lipídica a 20\% p/v & 2,0 & 4,2 & 8,5 & 12,5 & 6,0 & 10,0 & 15,0 \\
\hline Cloreto de sódio a $20 \%$ p/v & 0,8 & 0,8 & 0,8 & 0,8 & 1,2 & 1,2 & 1,2 \\
\hline Cloreto de potássio a $19,1 \%$ p/v & 0,4 & 0,4 & 0,4 & 0,4 & 0,8 & 0,8 & 0,8 \\
\hline Gluconato de cálcio a $10 \% \mathrm{p} / \mathrm{v}$ & 3,9 & 3,9 & 3,9 & 3,9 & 3,9 & 3,9 & 3,9 \\
\hline Sulfato de magnésio a $20 \% \mathrm{p} / \mathrm{v}$ & 0,1 & 0,1 & 0,1 & 0,1 & 0,2 & 0,2 & 0,2 \\
\hline Fosfato dibásico de potássio $1 \mathrm{mmol} / \mathrm{mL}^{* *}$ & 1,0 & 1,0 & 1,0 & 1,0 & 1,2 & 1,2 & 1,2 \\
\hline Oligoelementos (pediátrico) & 0,4 & 0,4 & 0,4 & 0,4 & 0,3 & 0,3 & 0,3 \\
\hline Água destilada q.s.p. & 100,0 & 100,0 & 100,0 & 100,0 & 100,0 & 100,0 & 100,0 \\
\hline
\end{tabular}


Nenhuma das formulações em sistema lipídico sem fosfato apresentou alterações macroscópicas visíveis, porém, quando avaliadas microscopicamente, observou-se, antes da filtração, 30 minutos após o preparo e nos tempos subseqüentes, a presença de uma grande quantidade de gotículas de gordura agregadas (Figuras 1-C), significativamente maiores que as hemácias (Figuras 1-D). No tempo zero, após a filtração, essa ocorrência não foi observada. Entretanto, após 30 minutos, observou-se a presença de gotículas de gordura aumentadas e número maior após 02 horas e 24 horas. A Figura 1-E mostra o aspecto microscópico de uma emulsão lipídica sem alteração.

Nas formulações em sistema lipídico com fosfato, observou-se, antes da filtração, no tempo $30 \mathrm{mi}$ nutos, a presença de gotículas de gordura aumentadas, agregadas e de precipitados de fosfato de cálcio (Figura 1-F). Essas gotículas de gordura e os precipitados foram eliminados após a filtração, com filtros cujos poros foram de 1,2 $\mu \mathrm{m}$. Entretanto, 30 minutos após a filtração, novas gotículas de gordura aumentadas já estavam presentes. Após duas horas, detectaram-se cristais de fosfato de cálcio e um grande número de gotículas de gordura aumentadas e agregadas. Após 24 horas, as alterações estavam significativamente aumentadas. Nos filtros, foram encontrados retidos os precipitados de fosfato de cálcio.

\section{DISCUSSÃO}

Em resumo, os resultados do presente trabalho mostraram a presença de cristais amorfos e/ou cristalinos, além de agregados lipídicos, muitas vezes de tamanho superior a hemácias. Dessa maneira, esses resultados evidenciaram claramente que o uso de soluções contendo sais de fosfato inorgânico juntamente com sais de cálcio orgânico ou inorgânico, adicionados às nutrições parenterais, contendo ou não emulsões lipídicas, deve ser feito com extremo rigor. Em geral, a precipitação desses sais ocorre quando uma das fontes desses minerais é inorgânica ${ }^{(11)}$. No futuro, espera-se solucionar esse problema com a utilização de sais orgânicos, como o glicerofosfato de sódio/potássio como fonte de fósforo. Entretanto, a evidência clínica da necessidade de incorporação de micronutrientes a essas formulações é sempre motivo de preocupação, pois implicará na utilização de novos sais inorgânicos ou orgânicos, que poderão aumentar a probabilidade de instabilização das nutrições parenterais.
Os resultados, aqui apresentados, também demonstraram as dificuldades na estabilização de nutrições parenterais contendo lipídios em solução aquo$\mathrm{sa}^{(13)}$. A título de comparação, o tamanho máximo do quilomícron oscila em torno de $1,2 \mu \mathrm{m}$ e o diâmetro médio da hemácia é de 7,5 $\mu \mathrm{m}$. Não se recomenda a infusão intravenosa de emulsões lipídicas contendo gotículas de gordura com dimensões iguais ou superiores a $05 \mu \mathrm{m}$. Agregados de lipídios, com diâmetros superiores a esse último valor, podem embolizar-se no leito capilar pulmonar de pacientes recebendo nutrição parenteral contendo lipídios ${ }^{(12)}$. Com a finalidade de se evitar esse problema, a American Society for Parenteral and Enteral Nutrition (ASPEN), por sugestão do Food and Drug Administration (FDA), recomenda que se utilizem filtros com poros de $0,22 \mu \mathrm{m}$ para as soluções sem lipídios, e de 1,2 $\mu \mathrm{m}$, quando se utilizar nutrição parenteral com lipídios. Alternativamente, os filtros de $1,2 \mu \mathrm{m}$ podem ser utilizados para ambas as nutrições parenterais.

É responsabilidade do Serviço de Farmácia de um determinado hospital e, em última análise, do farmacêutico responsável pela preparação das nutrições parenterais a utilização de meios que evitem precipitação e instabilidade. Para tal, o farmacêutico deve verificar e analisar as formulações, escolher corretamente os fármacos nutricionalmente corretos e condições ideais de preparo.

\section{Por fim, considerando-se que:}

1) há possibilidade de precipitação, quando da utilização de sais inorgânicos, em especial o fosfato dibásico de sódio, ou potássio como fonte de fósforo, com gluconato de cálcio como fonte de cálcio;

2) há formação de agregados e/ou crescimento das partículas de lipídios, em especial quando se altera o meio pela adição de sais inorgânicos divalentes;

3) a filtração, imediatamente após o preparo da nutrição parenteral, não elimina os riscos da formação de novos precipitados de fosfato de cálcio e agregados de gotículas de gordura, verifica-se que não foi confirmada a hipótese, e recomenda-se, portanto, o uso de filtros com poros de $1,2 \mu \mathrm{m}$ de diâmetro durante a infusão de nutrições parenterais, com a finalidade de se promover uma barreira mecânica, que impossibilite a passagem para a corrente sangüínea de precipitados e de gotículas de gordura aumentadas e/ou agregadas, indesejáveis a essas nutrições. 


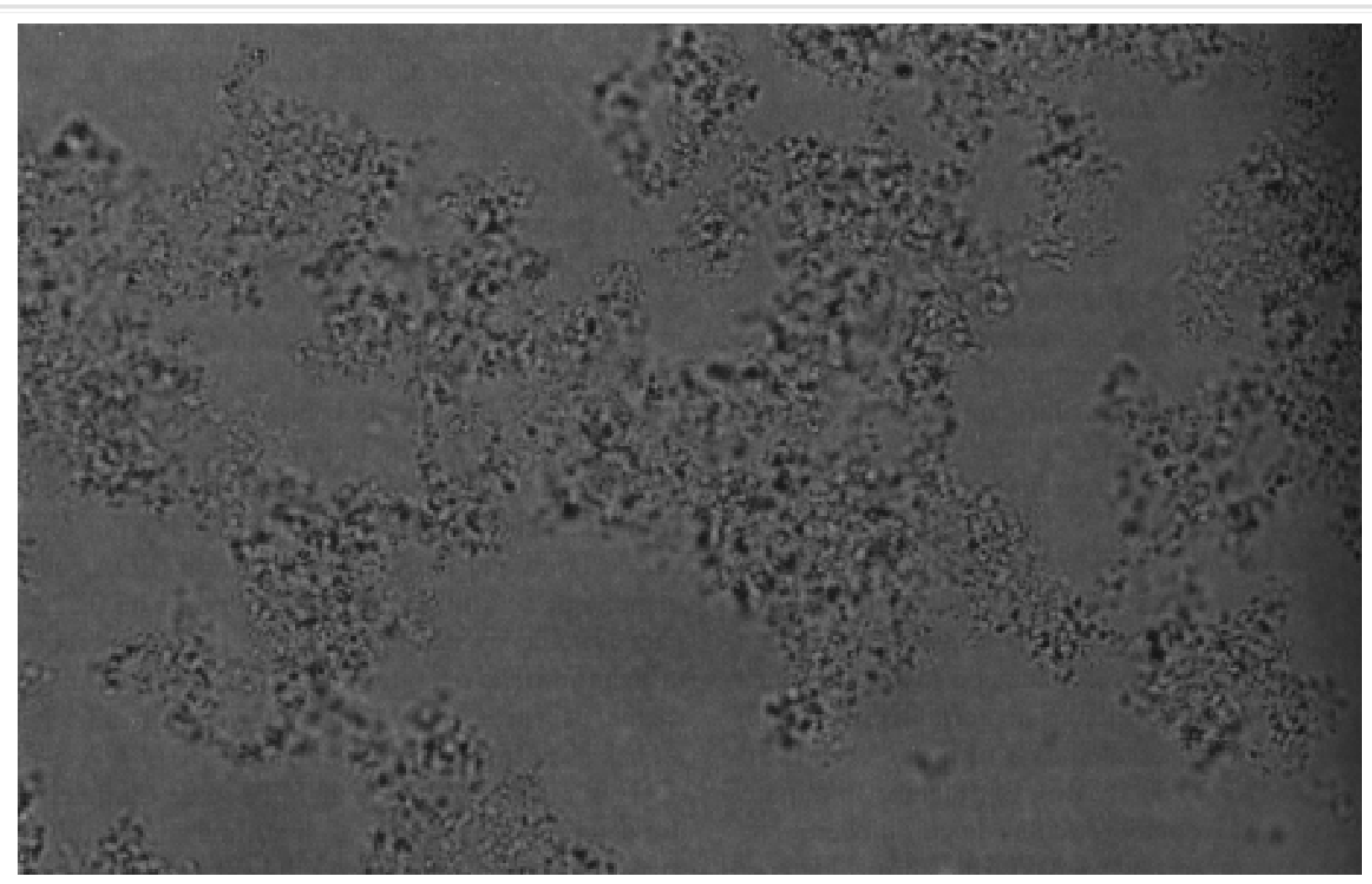

Figura 1A - Fosfato de Cálcio Amorfo.

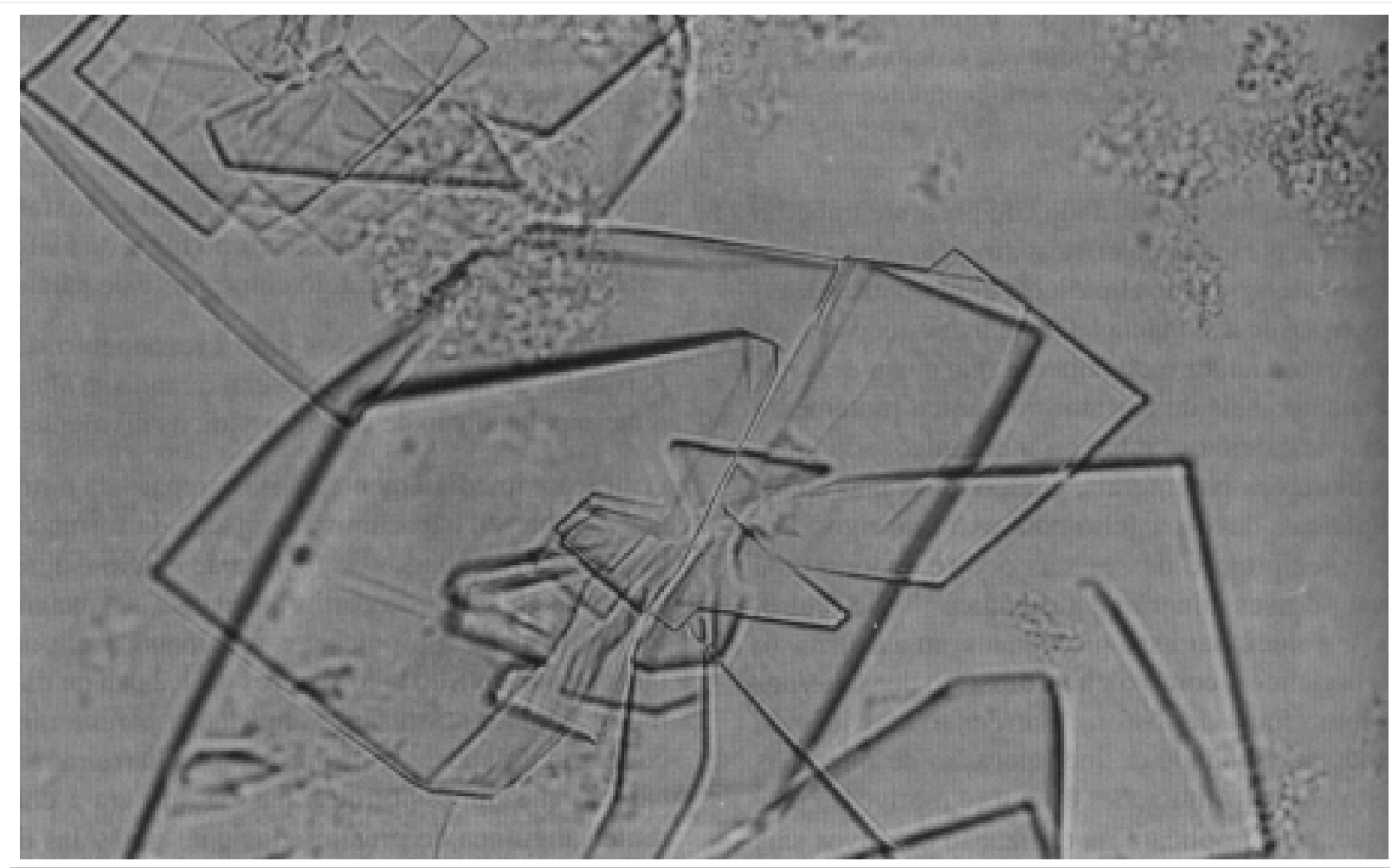

Figura 1B - Fosfato de Cálcio - Cristais. 


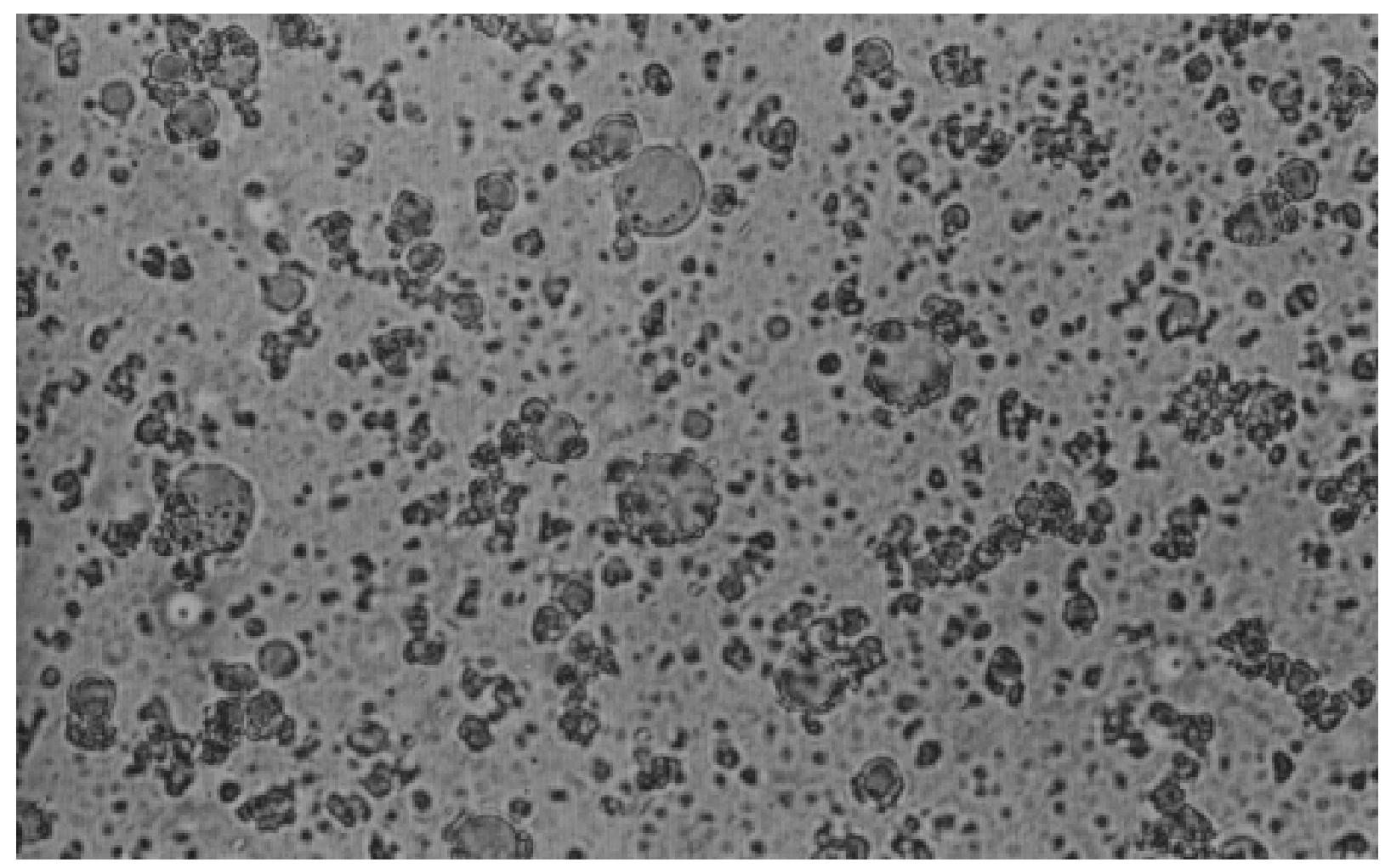

Figura 1C - Gotículas de Gordura Aumentadas.

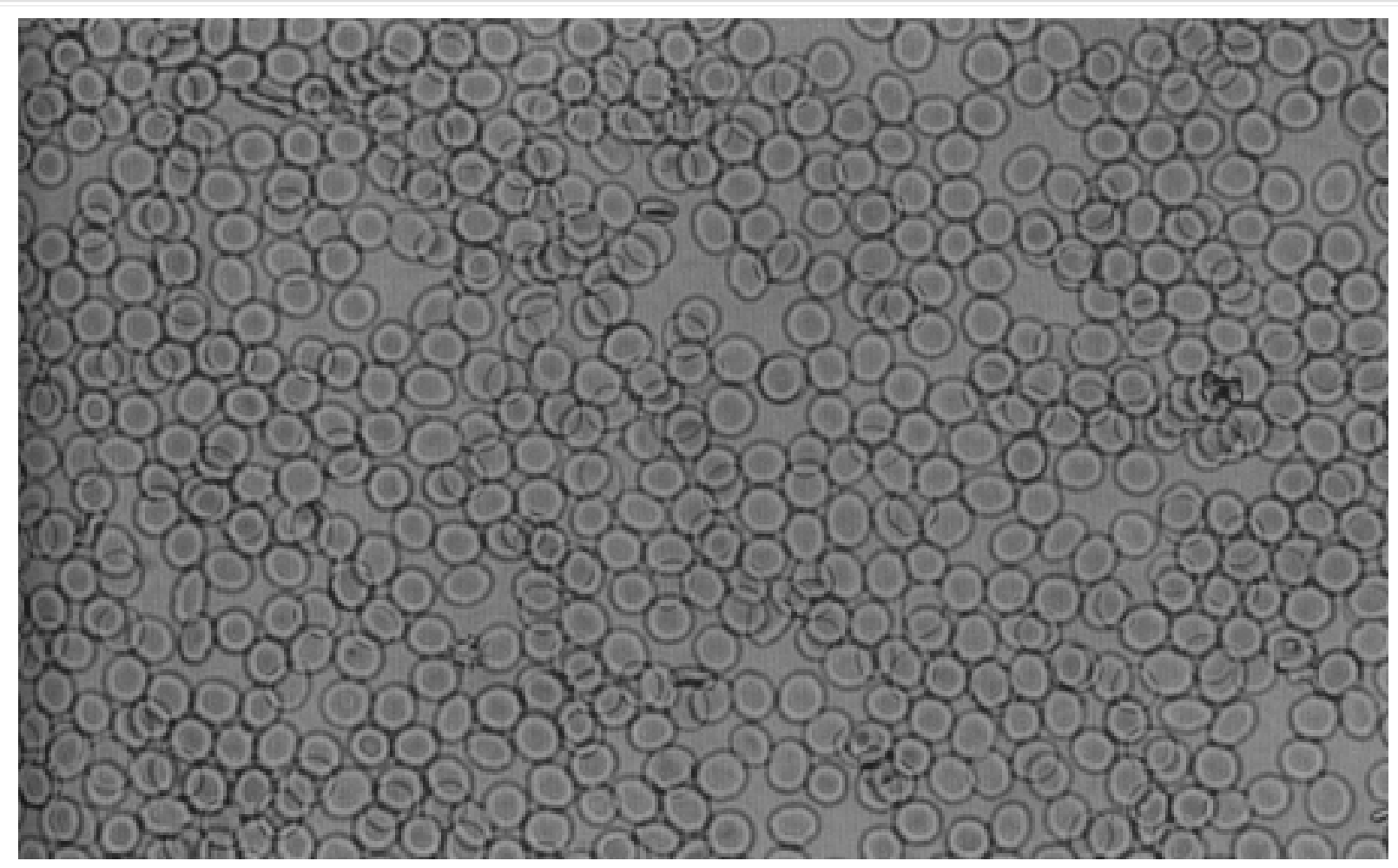

Figura 1D - Hemácias, tamanho médio 7,5 $\mu \mathrm{m}$. 


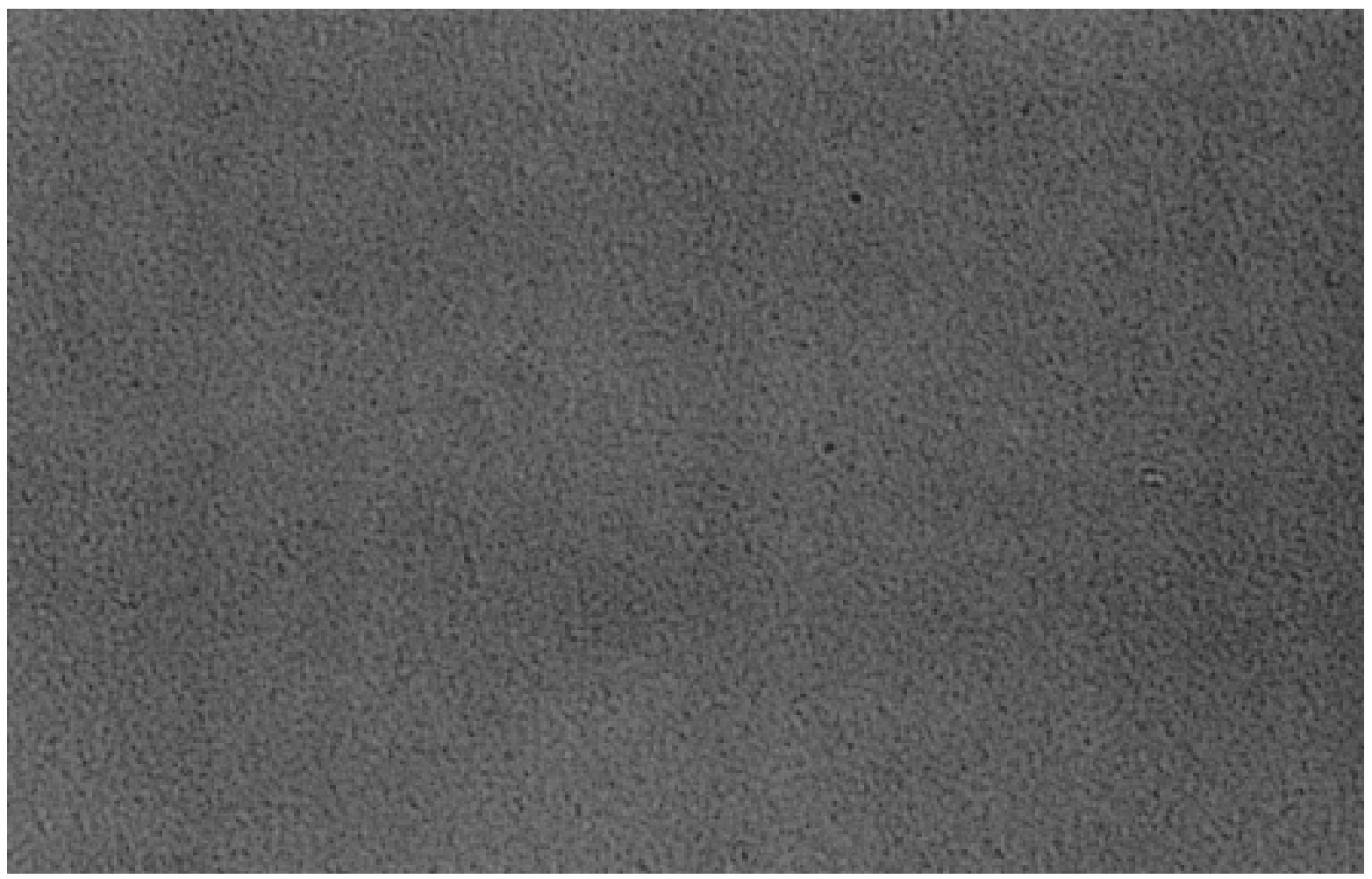

Figura 1E - Gotículas de Gordura, tamanho médio 0,45 $\mu \mathrm{m}$.

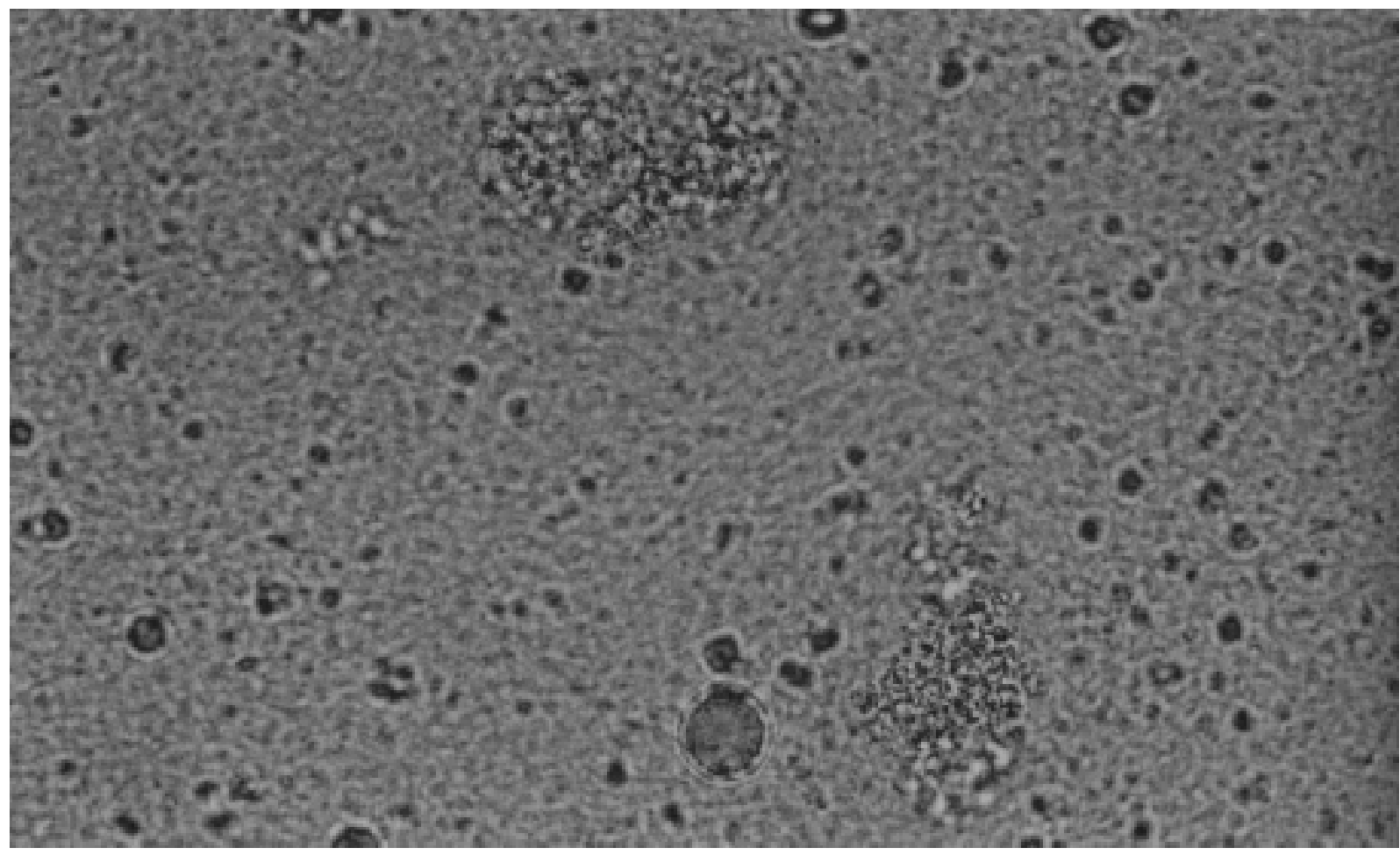

Figura 1F - Gotículas e gordura aumentadas e precipitados de fosfato de cálcio. 
SAKAMOTO LM; ALCÂNTARA ST; PASSOS NMRRS; HOTTA JKS \& MARCHINI JS. The importance of the use of filters during the infusion of parenteral nutrition containing lipid emulsions. Medicina, Ribeirão Preto, 32: 478-485, oct./dec. 1999.

ABSTRACT: Parenteral nutrition preparations supplemented with lipid emulsions may present physicochemical alterations characterized by the formation of amorphous or crystalline precipitates and by an increased size of fat droplets, which aggregate. A macroscopic verification of these changes is not always possible because of the opaque aspect of the emulsions.

The objective of the present in vitro study was to determine the presence of calcium phosphate precipitates, of fat droplets of the lipid emulsion with wider diameters than red blood cells in parenteral nutrition preparations and the importance of the use of filters for the retention of such precipitates. These events were demonstrated by macroscopic and microscopic inspection of parenteral nutrition preparations containing potassium dibasic phosphate and calcium gluconate as a source of phosphorus and calcium, respectively, added to the mixture of amino acid solutions at the proportion of $10 \%(\mathrm{w} / \mathrm{v})$, with $50 \%$ glucose $(\mathrm{w} / \mathrm{v})$, a $20 \%$ lipid emulsion $(\mathrm{w} / \mathrm{v})$ and minerals at different proportions. The results showed the presence of a calcium precipitate and of enlarged and/or aggregated fat droplets before filtration, and their absence immediately after filtration. Conclusion:the use of filters with $1.2 \mu \mathrm{m}$ pores was sufficient to retain the precipitates and to prevent the passage of fat droplets with dimensions that might cause microvascular embolism in patients submitted to parenteral nutrition.

UNITERMS: Filtration. Parenteral Nutrition. Emulsions.

\section{REFERÊNCIAS BIBLIOGRÁFICAS}

1 - RIELLA MC. Suporte nutricional parenteral e enteral. $2^{\mathrm{a}}$ ed. Guanabara Koogan, Rio de Janeiro, 1993.

2 - TANNURI U \& TELLES JR M. Suporte nutricional em pediatria. Atheneu Editora, São Paulo, 1994.

3 - CUNHA SFC \& CUNHA DF. Suporte nutricional. In: OLIVEIRA, JEDO \& MARCHINI JS, eds. Ciências nutricionais. Sarvier Editora, São Paulo, p. 289-303, 1998.

4 - MARCHINI JS; OKANO N; CUPO P; PASSOS NMRRS; SAKAMOTO LM \& BASILE-FILHO A. Nutrição parenteral princípios gerais, formulários de prescrição e monitorização. Medicina, Ribeirão Preto 31: 62-72, 1998.

5 - BRASIL. Portaria № 272, de 08 de abril de 1998. Secretaria de Vigilância Sanitária. Ministério da Sáude, Brasília, 1998.

6 - MIRTALLO SM. The complexity of mixing calcium and phosphate. Promoting rational drug use through the ASHP Commission on Therapeutics. Am J Hosp Pharm 51: 15341536, 1994.

7 - DRISCOLL DF; BHARGAVA HV; LI L; ZAIM RH; BABAYAN VK \& BISTRIAN BR. Physicochemical stability of total nutrient admixtures. Am J Healthh Syst Pharm 52: 623-634, 1995.
8 - MACMAHON P; MAYNE PD; BLAIR M; POPE C \& KOVAR IZ. Calcium and phosphorus solubility in neonatal intravenous feeding solutions. Arch Dis Child 65: 352-353, 1990.

9 - EGGERT LD; RUSHO WJ; MACKAY MW \& CHAN GM. Calcium and phosphorus compatibility in parenteral nutrition solutions for neonates. Am J Hosp Pharm 39: 49-53, 1982.

10 - MACKAY MW; FITZGERALD KA \& JACKSON D. The solubility of calcium and phosphate in two specialty amino acid solutions. JPEN J Parenter Enteral Nutr 20:63-66, 1996.

11 - DRISCOLL DF; NEWTON DW \& BISTRIAN BR. Precipitation of calcium phosphate from parenteral nutrient fluids. Am J Hosp Pharm 51: 2834-2836, 1994.

12 - LUMPKIN MM \& BURLINGTON DB. Safety alert: hazards of precipitation associated with parenteral nutrition. Food and Drug Administration. Am J Hosp Pharm 51: 1427-1428, 1994.

13 - BROWN RD; QUERCIA RA \& SIGMAN R. Total nutrient admisture: a review. JPEN J Parenter Enteral Nutr 10: 650-658, 1986.

Recebido para publicação em 06/07/1999

Aprovado para publicação em 28/12/1999 